\title{
Is D2 Lymphadenectomy Essential for Cytology-positive Gastric Cancer? A Retrospective Analysis
}

\author{
SHUNJI ENDO ${ }^{1}$, KAZUHIRO NISHIKAWA ${ }^{2}$, KAZUMASA FUJITANI ${ }^{3}$, JIN MATSUYAMA ${ }^{4}$, \\ MASAKAZU IKENAGA ${ }^{4}$, TERUMASA YAMADA ${ }^{4}$, SHIGEYUKI TAMURA ${ }^{1}$ and YO SASAKI ${ }^{1}$ \\ ${ }^{1}$ Department of Surgery, Yao Municipal Hospital, Osaka, Japan; \\ ${ }^{2}$ Department of Surgery, National Hospital Organization Osaka National Hospital, Osaka, Japan; \\ ${ }^{3}$ Department of Gastroenterological Surgery, Osaka General Medical Center, Osaka, Japan; \\ ${ }^{4}$ Department of Gastroenterological Surgery, Higashiosaka City Medical Center, Osaka, Japan
}

\begin{abstract}
Background: The appropriate extent of lymphadenectomy on gastric cancer patients with positive peritoneal cytology (CY1) is uncertain. This study retrospectively compared overall survival (OS) after standard and limited lymphadenectomy. Patients and Methods: The medical records from four institutions from 2004 to 2018 were reviewed and data for 91 patients with CY1, but no other distant metastases, who underwent gastrectomy were analyzed. D2 or greater lymphadenectomy and less than D2 lymphadenectomy were performed in 51 and 40 patients, respectively. Results: Full cohort analyses showed that patients who underwent D2 or greater lymphadenectomy had better prognostic nutritional indices and more such patients received postoperative chemotherapy. The OS in the group treated with D2 or greater lymphadenectomy was also significantly better $(p=0.045)$. Twenty-seven pairs of patients were generated via propensity score matching, and analysis of their OS showed no significant difference between the groups according to lymphadenectomy ( $p=0.61)$. Conclusion: The extent of lymphadenectomy may not affect the prognoses for patients with CYI gastric cancer.
\end{abstract}

Gastric cancer was estimated to be the fourth most common cause of cancer death worldwide, behind lung, liver, and colorectal cancer, in 2016 (1). Although early gastric cancer can be cured by endoscopic resection or surgery, the prognosis of advanced gastric cancer with distant metastases is still poor. The presence of free cancer cells in the abdominal cavity, which can be determined by peritoneal lavage cytology from the pouch of Douglas during surgery, is classified as CY1 in

Correspondence to: Shunji Endo, MD, Ph.D., Department of Surgery, Yao Municipal Hospital, 1-3-1 Ryugecho, Yao, 581-0069 Osaka, Japan. Tel: +81 729220881, Fax: +81 729228167, e-mail: shunji.endo@hosp-yao.osaka.jp

Key Words: Cell biology, lymph node excision, stomach neoplasms. the Japanese classification of gastric carcinoma (2), is included in distant metastasis, and is categorized as stage IV in both the Japanese classification and the International Union Against Cancer TNM classification (3). In the Japanese gastric cancer registry in 2010 (4), the 5-year overall survival (OS) rates for patients with CY0 and CY1 gastric cancer were $66.2 \%$ and $13.1 \%$, respectively.

The CCOG0301 study, a phase II study of radical surgery followed by postoperative chemotherapy with S-1 for CY1 gastric cancer, showed excellent outcomes with a median survival time (MST) of 23.1 months and a 5-year survival rate of $26 \%$ (5). In that study, gastrectomy was performed with D2 lymphadenectomy. Since that study, radical gastrectomy with D2 lymphadenectomy has been recognized as a standard therapy for CY1 gastric cancer without other distant metastases. However, in clinical practice, limited lymphadenectomy of D1+, D1, or D0 has often been performed for CY1 gastric cancer $(6,7)$. Because recurrences after radical gastrectomy in patients with CY1 disease were most frequently found in the peritoneum (8), radical lymphadenectomy did not seem to be associated with survival benefit. D1+ or lesser lymphadenectomy may be appropriate for CY1 gastric cancer (6). However, the optimal lymphadenectomy for CY1 gastric cancer has not yet been clarified.

In this study, we hypothesized that limited lymphadenectomy was not inferior to standard lymphadenectomy in terms of OS. We retrospectively analyzed and compared the survival of each group by using propensity score matching (PSM).

\section{Patients and Methods}

Patients. We retrospectively reviewed the medical records from four institutions between 2004 and 2018 of 109 consecutive patients with CY1 gastric cancer, but no other distant metastases, such as peritoneal, liver, or extraregional lymph node metastases. Of these, 91 patients who underwent total or distal gastrectomy without any macroscopic residual tumor were analyzed. The 
following clinical and pathological characteristics, perioperative treatment, and postoperative outcomes were collected: Sex, age, Eastern Cooperative Oncology Group Performance Status (PS) (9), preoperative Onodera's prognostic nutritional index (PNI) (10), operative procedure, extent of lymph node dissection, macroscopic tumor type, histological classification, clinical and pathological tumor invasion (cT, pT), clinical and pathological lymph node metastasis $(\mathrm{cN}, \mathrm{pN})$, pre- and postoperative chemotherapy, first site of recurrence, and prognosis. The clinicopathological findings of gastric cancer were described according to the third English edition of the Japanese classification of gastric carcinoma (2). The PNI was calculated using the following formula: $10 \times$ serum albumin $(\mathrm{g} / \mathrm{dl})+0.005 \times$ total lymphocyte count $\left(/ \mathrm{mm}^{3}\right)(10)$.

Operative procedure. In principle, all surgical procedures were performed according to the Japanese gastric cancer treatment guidelines (11). Peritoneal lavage cytology was performed routinely immediately after laparotomy and before manipulation of the primary tumor. Ascites was aspirated if present, and $100 \mathrm{ml}$ of physiological normal saline was instilled and aspirated from the pouch of Douglas if ascites was absent. The presence of carcinoma cells on peritoneal lavage cytology were defined as CY1. At the participating institutions, cytological examination results are available during surgery, usually before supra-pancreatic lymph node dissection. For CY1, standard or limited lymphadenectomy was chosen based on the surgeon's policy and the most recent trend. The extent of lymphadenectomy was defined according to the fourth edition of the Japanese gastric cancer treatment guidelines as follows (11). For total gastrectomy: D0: lymphadenectomy less than D1; D1: dissection of lymph node stations 1-7; D1+: dissection of lymph node stations 1-7, 8a, 9 and $11 \mathrm{p}$; and D2: dissection of lymph node stations 1-7, 8a, 9, 10,11p, $11 \mathrm{~d}$ and 12a. For distal gastrectomy: D0: lymphadenectomy less than D1; D1: dissection of lymph node stations 1, 3, 4sb, 4d, 5, 6, and 7; D1+: dissection of lymph node stations 1, 3, 4sb, 4d, 5, 6, 7, 8a and 9; and D2: dissection of lymph node stations 1, 3, 4sb, 4d, 5, 6, 7, 8a, 9, 11p and 12a. Extended lymphadenectomy beyond D2 is defined as D2+. Lymphadenectomy not complying with the D2 criteria was classified as $<$ D2. Splenectomy can be omitted for total gastrectomy with D2 lymphadenectomy unless the tumor is located in the greater curvature of the upper stomach (12). Dissection of the lymph node station 12a for total gastrectomy was also optional because the 13th edition of the Japanese classification of gastric carcinoma (13), used between June 1999 and May 2010, excluded this lymph node station from D2. Laparoscopic surgery was not performed in the current series.

Propensity score matching. PSM was performed to minimize the effects of confounding biases and to adjust for significant differences in baseline characteristics between the standard and limited lymphadenectomy groups. The propensity scores of the patients were estimated using a logistic regression model. One-toone pair matching was performed without replacement by using a 0.20 caliper. The variables used in the PSM were selected based on the univariate analyses.

Statistical analysis. Continuous variables were compared using the Mann-Whitney $U$-test. Categorical variables were compared using the chi-square test with/without Yates' correction or Fisher's exact probability test. Survival was evaluated on Kaplan-Meier curves and compared using the log-rank test. OS was defined as the interval from the date of surgery to the date of death from any cause. Univariate and multivariate analyses for OS were performed using Cox proportional hazards model. All reported $p$-values were two-sided. Statistical significance was determined by $p<0.05$. All analyses were performed using JMP software (version 11.0.0 for Windows; SAS Institute Inc., Cary, NC, USA).

Disclosure of ethical statement. The protocol for this research project was approved by suitably constituted Ethics Committees of the Institutions, and conforms to the provisions of the Declaration of Helsinki: Committee of YMH, approval no. H30-75; committee of NHOONH, approval no. 19012; committee of OGMC, approval no. 30-S15-002; and committee of HCMC, approval no. 02-0409.

\section{Results}

Of the 91 patients with CY1, but no other distant metastases, who underwent gastrectomy, 48 underwent total gastrectomy and 43 patients underwent distal gastrectomy. In the current study, 51 patients who underwent D2 or D2+ lymphadenectomy were classified into the standard lymphadenectomy group, and 40 patients who underwent D0, D1, or D1+ lymphadenectomy were classified into the limited lymphadenectomy group $(<\mathrm{D} 2$ group). At the time of analysis, 81 patients (89\%) had died, and the median follow-up period of the 10 surviving patients was 109 months. Recurrences were detected by computed tomography scan in 57 patients $(63 \%)$.

Full cohort analyses. Table I shows the comparison of the clinical, surgical, and pathological characteristics of the standard lymphadenectomy and <D2 groups. In the standard lymphadenectomy group, the patients had significantly better PNIs, and significantly more of them received postoperative chemotherapy than those in the $<\mathrm{D} 2$ group.

The OS curves of the patients in the two groups are shown in Figure 1. The MST of the standard lymphadenectomy and $<$ D2 groups was 21.9 and 13.1 months, respectively $(p=0.045)$. The 1-, 3-, and 5-year survival rates were $78.4 \%$, $28.2 \%$, and $18.1 \%$ for the standard lymphadenectomy group and $55.0 \%, 20.0 \%$, and $10.0 \%$ for the $<\mathrm{D} 2$ group, respectively. In the standard lymphadenectomy group, 31 and two patients died of gastric cancer and other disease, respectively, among 35 patients whose causes of death were known (Table II). On the other hand, in the $<\mathrm{D} 2$ group, 21 and nine patients died of gastric cancer and other disease, respectively, among 32 patients whose causes of death were known. Significantly more patients died of other disease in the $<\mathrm{D} 2$ group than in the standard lymphadenectomy group $(p=0.007)$. The site of first recurrence was the peritoneum in $23(45 \%)$ and 17 patients $(43 \%)$ and lymph nodes in seven $(14 \%)$ and five patients $(13 \%)$ for the standard and <D2 lymphadenectomy groups, respectively, without any statistically significant difference (Table III). 
Table I. Comparison of the clinical, surgical, and pathological features of the standard $(\geq D 2)$ and limited $(<D 2)$ lymphadenectomy groups in the full cohort.

\begin{tabular}{|c|c|c|c|c|}
\hline \multicolumn{2}{|l|}{ Variable } & \multirow{2}{*}{$\frac{\geq \mathrm{D} 2(\mathrm{n}=51)}{39(76)}$} & \multirow{2}{*}{$\frac{<\mathrm{D} 2(\mathrm{n}=40)}{32(80)}$} & \multirow{2}{*}{$\frac{p \text {-Value }}{0.69}$} \\
\hline Gender, n (\%) & Male & & & \\
\hline Age, years & Median (IQR) & $71(62-74)$ & $72(66-81)$ & 0.14 \\
\hline \multirow{4}{*}{ PS score, $n$} & 0 & 38 & 24 & 0.17 \\
\hline & 1 & 6 & 8 & \\
\hline & 2 & 4 & 5 & \\
\hline & 3 & 3 & 3 & \\
\hline PNI & Median (IQR) & $44.0(39.0-49.0)$ & $37.8(33.3-46.0)$ & 0.005 \\
\hline Preoperative chemotherapy, $\mathrm{n}(\%)$ & Yes & $9(18)$ & $5(13)$ & 0.50 \\
\hline \multirow[t]{5}{*}{$\mathrm{cT}, \mathrm{n}$} & 1 & 1 & 0 & 0.80 \\
\hline & 2 & 3 & 2 & \\
\hline & 3 & 21 & 14 & \\
\hline & $4 a$ & 23 & 20 & \\
\hline & $4 \mathrm{~b}$ & 3 & 4 & \\
\hline \multirow[t]{4}{*}{$\mathrm{cN}, \mathrm{n}$} & 0 & 13 & 16 & 0.13 \\
\hline & 1 & 16 & 10 & \\
\hline & 2 & 14 & 12 & \\
\hline & 3 & 8 & 2 & \\
\hline \multirow[t]{2}{*}{ Extent of gastrectomy, $\mathrm{n}$} & Total & 27 & 21 & 0.97 \\
\hline & Distal & 24 & 19 & \\
\hline \multirow[t]{5}{*}{ Extent of lymphadenectomy, $\mathrm{n}$} & D0 & 0 & 5 & $<0.0001$ \\
\hline & D1 & 0 & 17 & \\
\hline & D1+ & 0 & 18 & \\
\hline & D2 & 48 & 0 & \\
\hline & $\mathrm{D} 2+$ & 3 & 0 & \\
\hline \multirow[t]{6}{*}{ Macroscopic type, $\mathrm{n}$} & $0-\mathrm{I}$ & 0 & 1 & 0.64 \\
\hline & 1 & 1 & 0 & \\
\hline & 2 & 12 & 7 & \\
\hline & 3 & 13 & 14 & \\
\hline & 4 & 21 & 16 & \\
\hline & 5 & 4 & 2 & \\
\hline \multirow[t]{2}{*}{ Histological classification, $\mathrm{n}$} & Differentiated (pap/tub/muc/other) & $24(1 / 17 / 5 / 1)$ & $19(0 / 17 / 2 / 0)$ & 0.97 \\
\hline & Undifferentiated (por/sig) & $27(25 / 2)$ & $21(18 / 3)$ & \\
\hline \multirow[t]{5}{*}{$\mathrm{pT}, \mathrm{n}$} & $1 \mathrm{~b}$ & 0 & 1 & 0.110 \\
\hline & 2 & 1 & 0 & \\
\hline & 3 & 9 & 1 & \\
\hline & $4 \mathrm{a}$ & 35 & 34 & \\
\hline & $4 \mathrm{~b}$ & 6 & 4 & \\
\hline \multirow[t]{4}{*}{$\mathrm{pN}, \mathrm{n}$} & 0 & 2 & 3 & 0.87 \\
\hline & 1 & 4 & 2 & \\
\hline & 2 & 10 & 8 & \\
\hline & 3 & 35 & 27 & \\
\hline Postoperative chemotherapy, n (\%) & Yes & $43(84)$ & $23(58)$ & 0.004 \\
\hline
\end{tabular}

IQR: Interquartile range; PS: Eastern Cooperative Oncology Group Performance Status; PNI: Onodera's prognostic nutritional index. Clinicopathological findings were written according to the Japanese Classification of Gastric Carcinoma (2).

Univariate and multivariate analyses. Univariate and multivariate analyses were performed to identify prognostic factors for OS. In the univariate analyses, PS 1-3, PNI of $<40$, cN2-3, <D2 lymphadenectomy, undifferentiated histological classification, $\mathrm{pN} 3$, and absence of postoperative chemotherapy were significantly correlated with worse OS (Table IV). Multivariate analysis was conducted with these significant factors and revealed that undifferentiated histological classification and the absence of postoperative chemotherapy were independent risk factors for death.

PSM cohort analyses. Based on the abovementioned univariate analyses, the patients' propensity scores were estimated using a logistic regression model based on the following eight variables: Age, sex, PS score, PNI, cN, extent of gastrectomy, histological classification, and postoperative 


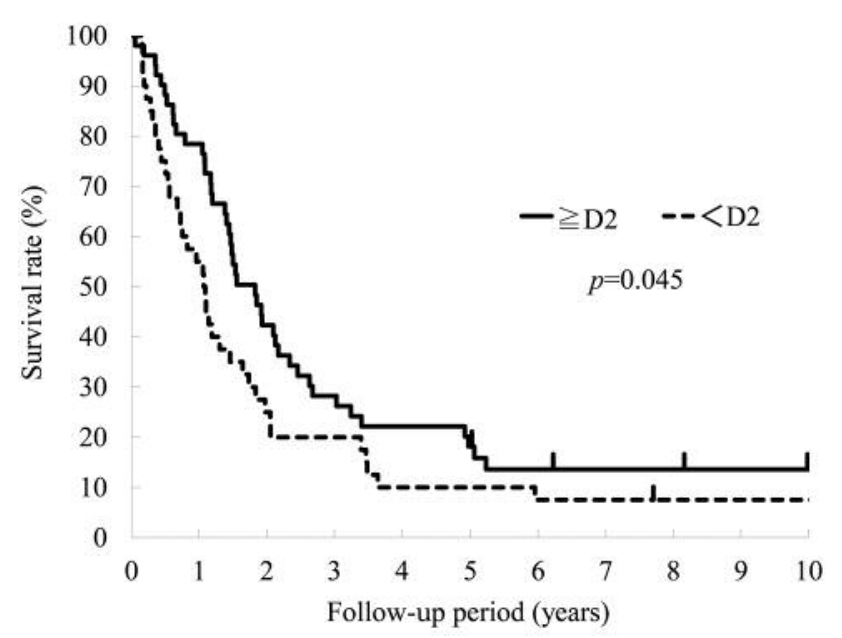

Figure 1. Kaplan-Meier overall survival curves of the standard $(\geq D 2)$ and $<D 2$ lymphadenectomy groups in the full cohort that underwent gastrectomy with CY1, but no other distant metastases.

Table II. Cause of death in the full cohort according to standard $(\geq D 2)$ and limited $(<D 2)$ lymphadenectomy.

\begin{tabular}{lccc}
\hline Variable, $\mathrm{n}$ & $\geq \mathrm{D} 2(\mathrm{n}=51)$ & $<\mathrm{D} 2(\mathrm{n}=40)$ & $p$-Value \\
\hline Gastric cancer & 31 & 21 & 0.43 \\
Surgical complication & 2 & 2 & 0.80 \\
Other disease & 2 & 9 & 0.007 \\
Pneumonia & 1 & 5 & \\
Cardiac failure & 0 & 2 & \\
Cerebral infarction & 0 & 1 & \\
Lung cancer & 0 & 1 & \\
Senility & 1 & 0 & \\
Unknown & 8 & 6 & \\
Alive & 8 & 2 & 0.11 \\
\hline
\end{tabular}

chemotherapy. Twenty-seven pairs of patients in the standard lymphadenectomy and $<\mathrm{D} 2$ lymphadenectomy groups were matched using the propensity scores. The patient characteristics of the two groups seemed to be well matched without any significant differences (Table V). Post-PSM comparisons of OS between the two groups are shown in Figure 2. The MST in the standard and $<\mathrm{D} 2$ lymphadenectomy groups was 21.9 versus 13.7 months, and $1-, 3-$, and 5-year OS rates of $77.7 \%, 18.5 \%$ and $14.8 \%$ versus $66.7 \%, 25.9 \%$ and $11.5 \%$, respectively $(p=0.61)$. The causes of death did not significantly differ between the two groups (Table VI). The site of first recurrence for the standard lymphadenectomy and $<\mathrm{D} 2$ lymphadenectomy groups were the peritoneum in $11(41 \%)$ and 13 patients (48\%) and the

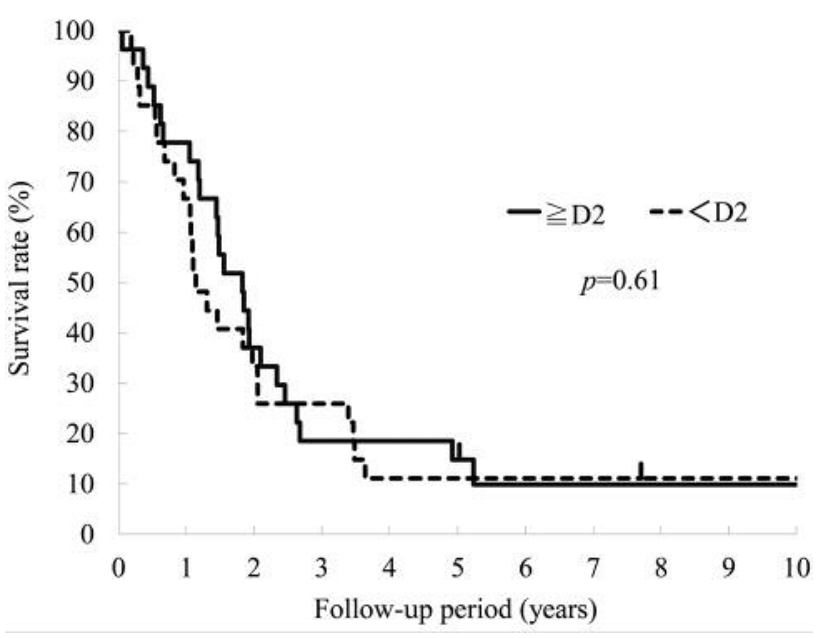

Figure 2. Kaplan-Meier overall survival curves of the standard $(\geq D 2)$ and $<D 2$ lymphadenectomy groups in the propensity score-matched cohort that underwent gastrectomy with CY1, but had no other distant metastases.

Table III. Site of first recurrence in the full cohort.

\begin{tabular}{lccc}
\hline Site & $\geq \mathrm{D} 2(\mathrm{n}=51)$ & $<\mathrm{D} 2(\mathrm{n}=40)$ & $p$-VaIue \\
\hline Peritoneum, n (\%) & $23(45 \%)$ & $17(43 \%)$ & 0.80 \\
Lymph node, n (\%) & $7(14 \%)$ & $5(13 \%)$ & 0.86 \\
Lung/pleura, n & 5 & 0 & 0.04 \\
Liver, n & 3 & 0 & 0.12 \\
Bone, n & 1 & 2 & 0.42 \\
None, n & 15 & 16 & \\
Unknown, n & 2 & 1 & \\
\hline
\end{tabular}

Data include duplication.

lymph nodes in three (11\%) and four patients (15\%), respectively, without any significant difference (Table VII).

\section{Discussion}

The present retrospective study showed that the prognoses for patients of the standard lymphadenectomy group were significantly better than those for the $<\mathrm{D} 2$ group in the full cohort analysis. However, the former group included patients with higher PNI; therefore, more patients in the former group received postoperative chemotherapy compared with the latter group. Postoperative chemotherapy was shown to be an independent prognostic factor for OS. As a result, the prognoses of the two groups were equivalent after PSM. The 
Table IV. Univariate and multivariate analyses for patients with CY1 disease without other distant metastases who underwent gastrectomy.

\begin{tabular}{|c|c|c|c|c|c|c|}
\hline \multirow[b]{2}{*}{ Variables } & \multirow[b]{2}{*}{$\mathrm{n}$} & \multirow[b]{2}{*}{ MST (months) } & \multicolumn{2}{|c|}{ Univariate analysis } & \multicolumn{2}{|c|}{ Multivariate analysis } \\
\hline & & & HR (95\%CI) & $p$-Value & HR (95\%CI) & $p$-Value \\
\hline \multicolumn{7}{|l|}{ Gender } \\
\hline Male & 71 & 17.4 & $0.93(0.57-1.61)$ & 0.80 & & \\
\hline Female & 20 & 19.5 & & & & \\
\hline \multicolumn{7}{|l|}{ Age } \\
\hline$\geq 70$ Years & 50 & 16.7 & $1.38(0.88-2.18)$ & 0.16 & & \\
\hline$<70$ Years & 41 & 17.8 & & & & \\
\hline \multicolumn{7}{|l|}{ PS } \\
\hline $1-3$ & 29 & 12.8 & $1.69(1.05-2.65)$ & 0.03 & $1.13(0.69-1.84)$ & 0.61 \\
\hline 0 & 62 & 19.7 & & & & \\
\hline \multicolumn{7}{|l|}{ PNI } \\
\hline$<40$ & 36 & 13.2 & $1.69(1.08-2.64)$ & 0.02 & $1.39(0.84-2.27)$ & 0.20 \\
\hline$\geq 40$ & 55 & 22.0 & & & & \\
\hline \multicolumn{7}{|l|}{$\mathrm{cT}$} \\
\hline 4 & 50 & 17.3 & $1.04(0.67-1.63)$ & 0.85 & & \\
\hline $1-3$ & 41 & 17.5 & & & & \\
\hline \multicolumn{7}{|l|}{$\mathrm{cN}$} \\
\hline $2-3$ & 36 & 9.5 & $1.68(1.06-2.64)$ & 0.03 & $1.49(0.92-2.37)$ & 0.10 \\
\hline $0-1$ & 55 & 13.0 & & & & \\
\hline \multicolumn{7}{|c|}{ Preoperative chemotherapy } \\
\hline No & 77 & 17.6 & $0.93(0.52-1.80)$ & 0.81 & & \\
\hline Yes & 14 & 13.4 & & & & \\
\hline \multicolumn{7}{|c|}{ Extent of gastrectomy } \\
\hline Total & 48 & 14.4 & $1.39(0.89-2.17)$ & 0.15 & & \\
\hline Distal & 43 & 19.7 & & & & \\
\hline \multicolumn{7}{|c|}{ Extent of lymphadenectomy } \\
\hline$<\mathrm{D} 2$ & 40 & 13.0 & $1.56(1.00-2.42)$ & 0.05 & $1.45(0.90-2.34)$ & 0.13 \\
\hline$\geq \mathrm{D} 2$ & 51 & 22.0 & & & & \\
\hline \multicolumn{7}{|l|}{ Macroscopic type } \\
\hline Type 4 & 37 & 16.9 & $1.39(0.88-2.16)$ & 0.15 & & \\
\hline Non-type 4 & 54 & 18.0 & & & & \\
\hline \multicolumn{7}{|c|}{ Histological classification } \\
\hline Undifferentiated & 48 & 13.7 & $1.72(1.10-2.70)$ & 0.02 & $2.16(1.32-3.58)$ & 0.002 \\
\hline Differentiated & 43 & 20.8 & & & & \\
\hline \multicolumn{7}{|l|}{ pT } \\
\hline 4 & 79 & 16.6 & $1.42(0.77-2.93)$ & 0.28 & & \\
\hline $1-3$ & 12 & 32.1 & & & & \\
\hline \multicolumn{7}{|l|}{$\mathrm{pN}$} \\
\hline 3 & 62 & 14.3 & $1.85(1.15-3.05)$ & 0.01 & & \\
\hline $0-2$ & 29 & 32.1 & & & & \\
\hline \multicolumn{7}{|c|}{ Postoperative chemotherapy } \\
\hline No & 25 & 6.5 & $2.82(1.72-4.51)$ & $<0.0001$ & $2.49(1.43-4.22)$ & 0.002 \\
\hline Yes & 66 & 22.0 & & & & \\
\hline
\end{tabular}

CI: Confidence intervaI; HR: hazard ratio; MST: median survival time; PS: Eastern Cooperative Oncology Group Performance Status; PNI: Onodera's prognostic nutritional index. Clinicopathological findings were written according to the Japanese Classification of Gastric Carcinoma (2).

site of first recurrence did not significantly differ after standard or $<\mathrm{D} 2$ lymphadenectomy.

According to the Japanese gastric cancer treatment guidelines (11), patients with $\mathrm{cN}+$ or cT2-4 are recommended to be treated with gastrectomy and D2 lymphadenectomy with curative intent. Globally, the consensus on D2 lymphadenectomy for gastric cancer surgery has been gathering support as a result of improved survival among patients after D2 lymphadenectomy and improvements in skill and experience using the D2 technique, as shown in some randomized controlled trials from the Netherlands (14), Taiwan (15), and Italy (16). Those trials may have included patients with CY1 disease, but subgroup data were unavailable. 
Table V. Comparison of the clinical, surgical, and pathological features of the standard $(\geq D 2)$ and limited $(<D 2)$ lymphadenectomy groups in the propensity score-matched cohort.

\begin{tabular}{|c|c|c|c|c|}
\hline \multicolumn{2}{|l|}{ Variable } & \multirow{2}{*}{$\frac{\geq \mathrm{D} 2(\mathrm{n}=27)}{21(78)}$} & \multirow{2}{*}{$\frac{<\mathrm{D} 2(\mathrm{n}=27)}{21(78)}$} & \multirow{2}{*}{$\begin{array}{c}p \text {-Value } \\
>0.99\end{array}$} \\
\hline Gender, n (\%) & Male & & & \\
\hline Age, years & Median (IQR) & $71(65-76)$ & $71(63-78)$ & 0.57 \\
\hline \multirow[t]{4}{*}{ PS score, $\mathrm{n}$} & 0 & 19 & 19 & 0.94 \\
\hline & 1 & 6 & 5 & \\
\hline & 2 & 1 & 2 & \\
\hline & 3 & 1 & 1 & \\
\hline PNI & Median (IQR) & $42.4(38.8-46.6)$ & $43.4(37.0-47.9)$ & 0.91 \\
\hline Preoperative chemotherapy, n (\%) & Yes & $3(11)$ & $4(15)$ & 0.69 \\
\hline \multirow[t]{5}{*}{$\mathrm{cT}, \mathrm{n}$} & 1 & 1 & 0 & 0.83 \\
\hline & 2 & 2 & 1 & \\
\hline & 3 & 9 & 9 & \\
\hline & $4 \mathrm{a}$ & 14 & 16 & \\
\hline & $4 \mathrm{~b}$ & 1 & 1 & \\
\hline \multirow[t]{4}{*}{$\mathrm{cN}, \mathrm{n}$} & 0 & 8 & 8 & $>0.99$ \\
\hline & 1 & 9 & 9 & \\
\hline & 2 & 8 & 8 & \\
\hline & 3 & 2 & 2 & \\
\hline \multirow[t]{2}{*}{ Extent of gastrectomy, $\mathrm{n}$} & Total & 13 & 15 & 0.59 \\
\hline & Distal & 14 & 12 & \\
\hline \multirow[t]{5}{*}{ Extent of lymphadenectomy, $\mathrm{n}$} & D0 & 0 & 5 & $<0.0001$ \\
\hline & D1 & 0 & 11 & \\
\hline & D1+ & 0 & 11 & \\
\hline & D2 & 26 & 0 & \\
\hline & $\mathrm{D} 2+$ & 1 & 0 & \\
\hline \multirow[t]{5}{*}{ Macroscopic type, $n$} & 1 & 1 & 0 & 0.26 \\
\hline & 2 & 6 & 3 & \\
\hline & 3 & 5 & 12 & \\
\hline & 4 & 14 & 11 & \\
\hline & 5 & 1 & 1 & \\
\hline \multirow[t]{2}{*}{ Histological classification, $\mathrm{n}$} & Differentiated (pap/tub/muc/other) & $12(1 / 7 / 3 / 1)$ & $12(0 / 11 / 1 / 0)$ & $>0.99$ \\
\hline & Undifferentiated (por/sig) & $15(13 / 2)$ & $15(12 / 3)$ & \\
\hline \multirow[t]{4}{*}{$\mathrm{pT}, \mathrm{n}$} & 2 & 1 & 0 & 0.53 \\
\hline & 3 & 3 & 1 & \\
\hline & $4 \mathrm{a}$ & 20 & 23 & \\
\hline & $4 \mathrm{~b}$ & 3 & 3 & \\
\hline \multirow[t]{4}{*}{$\mathrm{pN}, \mathrm{n}$} & 0 & 2 & 1 & 0.95 \\
\hline & 1 & 2 & 2 & \\
\hline & 2 & 6 & 6 & \\
\hline & 3 & 17 & 18 & \\
\hline Postoperative chemotherapy, n (\%) & Yes & $21(78)$ & $21(78)$ & $>0.99$ \\
\hline
\end{tabular}

IQR: Interquartile range; PS: Eastern Cooperative Oncology Group Performance Status; PNI: Onodera's prognostic nutritional index. Clinicopathological findings were written according to the Japanese Classification of Gastric Carcinoma (2).

For CY1 cases, surgery with curative intent is not indicated according to the treatment algorithm in the Japanese gastric cancer treatment guidelines (11). A chemotherapy-first strategy can be adopted if information regarding CY1 was available before surgery by staging laparoscopy (17). Although the survival benefit of non-curative gastrectomy (R2) in advanced gastric cancer with a non-curable factor confined to either the liver, peritoneum, or para-aortic lymph nodes was not shown by the REGATTA trial (18), no evidence has been published concerning the survival benefit of non-curative gastrectomy
(R1) in patients with CY1 disease without other distant metastases. Currently in Japan, primary gastrectomy with D2 lymphadenectomy is considered to be the tentative standard therapy for CY1 gastric cancer without other residual disease based on the CCOG0301 study $(5,11)$.

The validity of D2 lymphadenectomy, as well as the survival benefit of gastrectomy, has also been unclear in patients with CY1 disease. In the REGATTA trial, gastrectomy was performed with D1 lymphadenectomy because it was a non-curative surgery (18). Even in CY1 
Table VI. Cause of death in the propensity score-matched cohort.

\begin{tabular}{lccc}
\hline Variable, $\mathrm{n}$ & $\geq \mathrm{D} 2(\mathrm{n}=27)$ & $<\mathrm{D} 2(\mathrm{n}=27)$ & $p$-VaIue \\
\hline Gastic cancer & 18 & 17 & 0.78 \\
Surgical complication & 1 & 2 & 0.55 \\
Other disease & 1 & 3 & 0.30 \\
$\quad$ Pneumonia & 1 & 2 & \\
$\quad$ Lung cancer & 0 & 1 & \\
Unknown & 4 & 3 & 0.64 \\
Alive & 3 & 2 & \\
\hline
\end{tabular}

Table VII. Site of first recurrence in the propensity score-matched cohort.

\begin{tabular}{lccc}
\hline Site & $\geq \mathrm{D} 2(\mathrm{n}=27)$ & $<\mathrm{D} 2(\mathrm{n}=27)$ & $p$-VaIue \\
\hline Peritoneum, n (\%) & $11(41 \%)$ & $13(48 \%)$ & 0.58 \\
Lymph node, n (\%) & $3(11 \%)$ & $4(15 \%)$ & 0.68 \\
Lung/pleura, n & 3 & 0 & 0.07 \\
Liver, n & 1 & 0 & 0.31 \\
Bone, n & 0 & 2 & 0.15 \\
None, n & 7 & 9 & \\
Unknown, n & 2 & 1 & \\
\hline
\end{tabular}

Data include duplication.

cancer, the extent of lymphadenectomy was not thought to affect prognosis because CY1 may be closely associated with peritoneal dissemination.

In our full-cohort analyses, the OS of the standard lymphadenectomy group was significantly longer than that of the $<$ D2 group. Of note, nine patients in the $<$ D2 group died of other diseases including pneumonia, and cardiac failure, while only two patients died of other disease in the standard lymphadenectomy group. This is possibly because surgeons avoided radical lymphadenectomy for high-risk patients with comorbidities who often had poor PS or poor PNI. After PSM, patient characteristics were similar between the groups, the cause of death did not significantly differ, and the survival curves of both groups almost overlapped.

Multivariate analysis showed that postoperative chemotherapy may play a more important role than lymphadenectomy for CY1 cases. Previous studies have also reported that the absence of postoperative chemotherapy, as well as poorer PS (17), poorer PNI (19), and massive lymph node metastases (7, 8, 19-23), were independent unfavorable predictors of survival among patients with CY1 gastric cancer (7). Our univariate analyses also showed that PS 1-3, PNI of $<40, \mathrm{cN} 2-3,<\mathrm{D} 2$ lymphadenectomy, undifferentiated histological classification, $\mathrm{pN} 3$, and absence of postoperative chemotherapy were prognostic factors, but the multivariate analysis showed that only undifferentiated histological classification and lack of postoperative chemotherapy were independent predictors.
In our study on the site of first recurrence, more than $40 \%$ of patients developed apparent peritoneal metastases that were detected by computed tomographic scan. The actual incidence rate of peritoneal metastasis is estimated to be much higher as most peritoneal metastases are difficult to detect by computed tomographic scan. In some cases, postoperative chemotherapy might have destroyed free cancer cells before their implantation to the peritoneum; however, in most cases, cancer cells may survive and then become implanted. Since floating cancer cells are seeded in the peritoneal cavity from the gastric serosa, the extent of lymphadenectomy may not influence peritoneal recurrence. On the other hand, only $14 \%$ and $13 \%$ of patients developed their first recurrence in the lymph nodes after standard and $<\mathrm{D} 2$ lymphadenectomy, respectively. Although nodal status is reported to be a risk factor for CY1 gastric cancer $(7,8,19-23)$, the extent of lymph node dissection did not affect the lymph node recurrence rate in this population. D1+ or even D1 lymphadenectomy may be sufficient for patients with CY1 gastric cancer.

The present study has several potential limitations. FirstIy, the quality of lymphadenectomy may have differed among institutions and surgeons because the cases were retrospectively collected from four institutions over 15 years. The differences between D2 and D1+ may be slight for some surgeons. Secondly, the number of patients was small, particularly after PSM. The comparison between 27 pairs of patients might have caused a statistically insignificant difference. Thirdly, this study was limited by its retrospective nature even after PSM. We estimated the patients' propensity scores based on the predictors selected by univariate analyses but confounding biases may still have existed. A randomized controlled trial should be conducted to confirm the current results. However, such a prospective study seems difficult as the number of patients with CY1 disease who undergo gastrectomy is decreasing because staging laparotomy and neoadjuvant chemotherapy are becoming widely performed in clinical practice in Japan.

In conclusion, patients with CY1 gastric cancer survived longer after standard lymphadenectomy than after limited lymphadenectomy. However, PSM analyses failed to show that standard lymphadenectomy contributed to prolonged survival compared with limited lymphadenectomy. Limited lymphadenectomy may be sufficient for this population. Postoperative chemotherapy may play a more important role in survival than lymphadenectomy.

\section{Conflicts of Interest}

The Authors have no financial conflicts of interest to disclose concerning this study.

\section{Authors' Contributions}

S. Endo wrote the protocol. S. Endo, K. Nishikawa, K. Fujitani, and J. Matsuyama gathered the data. S. Endo analyzed the data. All 
Authors were involved in the drafting, review, and approval of the article and the decision to submit for publication.

\section{References}

1 Health Statistics and Information Systems. Geneva, World Health Organization, 2018. Available at: https://www.who.int/ healthinfo/global_burden_disease/estimates/en/ )Last accessed on 13th September 2019)

2 Japanese Gastric Cancer Association: Japanese Classification of Gastric Carcinoma: Third English Edition. Gastric Cancer 14(2): 101-112, 2011. PMID: 21573743. DOI: 10.1007/s10120-0110041-5

3 Brierley JD, Gospodarowicz MK and Wittekind C: TNM Classification of Malignant Tumours, Eighth Edition. Oxford, Wiley Blackwell, 2017.

4 Japanese Gastric Cancer Association: The Japanese Gastric Cancer Registry in 2010. Tokyo, 2018. Available at: http://www.jgca.jp/entry/iganhtml/doc/2010_report.pdf (Last accessed on 13th September 2019)

5 Kodera Y, Ito S, Mochizuki Y, Kondo K, Koshikawa K, Suzuki N, Kojima H, Kojima T, Matsui T, Takase T, Tsuboi K, Fujiwara M, Nakao A and Chubu Clinical Oncology Group: A phase II study of radical surgery followed by postoperative chemotherapy with S-1 for gastric carcinoma with free cancer cells in the peritoneal cavity (CCOG0301 study). Eur J Surg Oncol 35(11): 1158-1163, 2009. PMID: 19328643. DOI: 10.1016/j.ejso.2009.03.003

6 Yamashita K, Ushiku H, Katada N, Hosoda K, Moriya H, Mieno $\mathrm{H}$, Kikuchi S, Hoshi K and Watanabe M: Reduced preoperative serum albumin and absence of peritoneal dissemination may be predictive factors for long-term survival with advanced gastric cancer with positive cytology test. Eur J Surg Oncol 41(10): 13241332, 2015. PMID: 26251341. DOI: 10.1016/j.ejso.2015.05.021

7 Kano Y, Kosugi S, Ishikawa T, Otani T, Muneoka Y, Sato Y, Hanyu T, Hirashima K, Bamba T and Wakai T: Prognostic significance of peritoneal lavage cytology at three cavities in patients with gastric cancer. Surgery 158(6): 1581-1589, 2015. PMID: 25958064. DOI: 10.1016/j.surg.2015.04.004

8 Saito H, Kihara K, Kuroda H, Matsunaga T, Tatebe S and Ikeguchi M: Surgical outcomes for gastric cancer patients with intraperitoneal free cancer cell, but no macroscopic peritoneal metastasis. J Surg Oncol 104(5): 534-537, 2011. PMID: 21618248. DOI: $10.1002 /$ jso. 21983

9 Common Toxicity Criteria, Version 2.0. Tokyo, Japanese Clinical Oncology Group, 1999. Available at: http://ctep.cancer.gov/ protocolDevelopment/electronic_applications/docs/ctcv20_4-30992.pdf (Last accessed on 13th September 2019)

10 Onodera T, Goseki N and Kosaki G: Prognostic nutritional index in gastrointestinal surgery of malnourished cancer patients. J Jpn Surg Society 85(9): 1001-1005, 1984. PMID: 6438478.

11 Japanese Gastric Cancer Association: Japanese gastric cancer treatment guidelines 2014 (ver. 4). Gastric Cancer 20(1): 1-19, 2017. PMID: 27342689. DOI: 10.1007/s10120-016-0622-4

12 Sano T, Sasako M, Mizusawa J, Yamamoto S, Katai H, Yoshikawa T, Nashimoto A, Ito S, Kaji M, Imamura $\mathrm{H}$, Fukushima N, Fujitani K and Stomach Cancer Study Group of the Japan Clinical Oncology Group: Randomized controlled trial to evaluate splenectomy in total gastrectomy for proximal gastric carcinoma. Ann Surg 265(2): 277-283, 2017. PMID: 27280511. DOI: $10.1097 /$ SLA.0000000000001814
13 Japanese Gastric Cancer Association: Japanese Classification of Gastric Carcinoma, the 13th Edition (in Japanese), Tokyo, Kanehara, 1999.

14 Songun I, Putter H, Kranenbarg EM, Sasako M and van de Velde CJ: Surgical treatment of gastric cancer: 15-Year follow-up results of the randomised nationwide Dutch D1D2 trial. Lancet Oncol 11(5): 439-449, 2010. PMID: 20409751. DOI: 10.1016/S14702045(10)70070-X

15 Wu CW, Hsiung CA, Lo SS, Hsieh MC, Chen JH, Li AF, Lui WY and Whang-Peng J: Nodal dissection for patients with gastric cancer: A randomised controlled trial. Lancet Oncol 7(4): 309-315, 2006. PMID: 16574546. DOI: 10.1016/S1470-2045(06)70623-4

16 Degiuli M, Sasako M, Ponti A, Vendrame A, Tomatis M, Mazza C, Borasi A, Capussotti L, Fronda G, Morino M and Italian Gastric Cancer Study Group: Randomized clinical trial comparing survival after D1 or D2 gastrectomy for gastric cancer. Br J Surg 101(2): 23-31, 2014. PMID: 24375296. DOI: 10.1002/bjs.9345

17 Mezhir JJ, Shah MA, Jacks LM, Brennan MF, Coit DG and Strong VE: Positive peritoneal cytology in patients with gastric cancer: Natural history and outcome of 291 patients. Ann Surg Oncol 17(12): 3173-3180, 2010. PMID: 20585870. DOI: 10.1245/s 10434-010-1183-0

18 Fujitani K, Yang HK, Mizusawa J, Kim YW, Terashima M, Han SU, Iwasaki Y, Hyung WJ, Takagane A, Park DJ, Yoshikawa T, Hahn S, Nakamura K, Park CH, Kurokawa Y, Bang YJ, Park BJ, Sasako M, Tsujinaka T and REGATTA study investigators: Gastrectomy plus chemotherapy versus chemotherapy alone for advanced gastric cancer with a single non-curable factor (REGATTA): A phase 3, randomised controlled trial. Lancet Oncol 17(3): 309-318, 2016. PMID: 26822397. DOI: 10.1016/S1470-2045(15)00553-

19 Endo S, Ikenaga M, Ohta K, Ueda M, Tsuda Y, Kato R, Itakura $\mathrm{H}$, Matsuyama J, Nishikawa K and Yamada T: Prognostic factors for cytology-positive gastric cancer. Surg Today 49(1): 56-64, 2019. PMID: 30167924. DOI: 10.1007/s00595-018-1704-1

20 Yamamoto M, Kawano H, Yamaguchi S, Egashira A, Minami K, Taguchi K, Ikeda Y, Morita M, Toh Y and Okamura T: Comparison of neoadjuvant chemotherapy to surgery followed by adjuvant chemotherapy in Japanese patients with peritoneal lavage cytology positive for gastric carcinoma. Anticancer Res 35(9): 4859-4863, 2015. PMID: 26254379.

21 Lee SD, Ryu KW, Eom BW, Lee JH, Kook MC and Kim YW: Prognostic significance of peritoneal washing cytology in patients with gastric cancer. Br J Surg 99(3): 397-403, 2012. PMID: 22101572. DOI: 10.1002/bjs.7812

22 Fukuchi M, Mochiki E, Ishiguro T, Ogura T, Sobajima J, Kumagai Y, Ishibashi $\mathrm{K}$ and Ishida $\mathrm{H}$ : Prognostic factors for gastric cancer with cancer cells in the peritoneal cavity. Anticancer Res 36(5): 2481-2485, 2016. PMID: 27127161.

23 Kano K, Aoyama T, Maezawa Y, Nakajima T, Ikeda K, Yamada T, Sato T, Oshima T, Rino Y, Masuda M, Ogata T, Cho H and Yoshikawa T: The survival and prognosticators of peritoneal cytology-positive gastric cancer patients who received upfront gastrectomy and subsequent S-1 chemotherapy. Int J Clin Oncol 22(5): 887-896, 2017. PMID: 28456896. DOI: $10.1007 / \mathrm{s} 10147-$ 017-1128-8

Received September 17, 2019

Revised September 26, 2019

Accepted October 1, 2019 